\title{
Solving quadratic fractional programming problem
}

\author{
Nejmaddin A. Suleiman, Maher A. Nawkhass* \\ Department of Mathematics, College of Education, University of Salahaddin-Erbil-Iraq \\ *Corresponding authorE-mail: maher.nawkhass@gmail.com
}

\begin{abstract}
In this paper the Quadratic fractional objective programming problem (QFPP) with linear constraints, has been defined and developed. The special case for this problem was solved by using the Wolfe's method and a modified simplex approach, by suggesting an algorithm for each method to solve the problem. The computer application for algorithms was tested on a number of numerical examples, consequently reliable results have been found.
\end{abstract}

Keywords: Modified Simplex method, QFPP, Wolfe's Method.

\section{Introduction}

Quadratic fractional problems (i.e. Ratio of objectives that have numerator and denominator) has attracted considerable research and interest since they have been utilized in production planning, financial and corporative planning, health care and hospital planning. Several methods to solve such problems are proposed in (2006), Beck, Ben-Tal and Teboulle [2] who found a global optimal solution for a quadratically constrained fractional quadratic Problem with applications to the regularized total least square. (2011), Amaral, Bomze and Judice [8] provided completely positive and copositive optimization formulations for the constrained fractional quadratic problem (CFQP) and standard fractional quadratic problem (StFQP). Moreover, Quadratic fractional programming problems with quadratic constraints have been addressed by Fukushima and Hayashi (2008) [6]. In addition (2012) Babul [3] he studies a technique for solving special type quadratic programming problems. Also in (2012) Borza, Sham and Saraj [4] studied and suggested Solving Linear Fractional Programming Problems with Interval Coefficients in the Objective Function. A New Approach.

In order to extend this work we will solve a special case of QFPP by using Wolfe's Method alongside with the modified simplex approach.

\section{Some basic definition related to this paper}

Definition 2.1: A positive definite symmetric matrix $A$ is a matrix which has all positive eigenvalue. Equivalently, a matric $A$ is positive definite if and only if $Q(x)>0$, for any $x \neq 0$. [5]

Definition 2.2: A positive semi-definite symmetric matrix $A$ is a matrix which have all eigenvalue are positive, with the remaining ones being zero. Equivalently, a matric $A$ is positive semi-definite if and only if $Q(x) \geq 0$, for any $x \neq 0$. [5]

\section{Quadratic programming}

If the optimization problem assumes the form max. $\mathrm{z}($ or min. $\mathrm{z})=\alpha+C^{T} x+x^{T} G x$ subject to:

$\mathrm{Ax}\left(\begin{array}{l}\leq \\ \geq \\ =\end{array}\right) \mathrm{b}$

$\mathrm{x} \geq 0$ 
Where $A=\left(a_{i j}\right)_{m \times n}$, matrix of coefficients, $\forall i=1,2, \ldots, m$ and $=1,2, \ldots, n$, $b=\left(b_{1}, b_{2}, \ldots, b_{m}\right)^{T}, x=\left(x_{1}, x_{2}, \ldots, x_{n}\right)^{T}, C^{T}=\left(c_{1}, c_{2}, \ldots, c_{n}\right)^{T}$,

and $G=\left(g_{i j}\right)_{n \times n}$ is a positive definite or positive semi-definite symmetric square matrix, moreover the constraints are linear and the objective function is quadratic. Such optimization problem is said to be a quadratic programming $(\mathrm{QP})$ problem. [1]

\section{Wolfe's method}

The Wolfe's method can be summarized as following.

Although, the Wolfe's method is not the first method that has been developed for solving quadratic programming problems (QPP), it is one of the most widely used methods for QPP and was developed by P. wolfe in 1959 [9]. Wolfe's algorithm can be directly applied to solve any quadratic programming problems of the form [7]

$\max z=f(x)=\sum_{j=1}^{n} c_{j} x_{j}+\frac{1}{2} \sum_{j=1}^{n} \sum_{k=1}^{n} c_{j k} x_{j} x_{k}, \quad$ subject to the constraints $\sum_{j=1}^{n} a_{i j} x_{j} \leq b_{j}, x_{j} \geq 0(i=$ $1,2, \ldots, m ; j=1,2, \ldots, n)$ where $c_{j k}=c_{k j}$ for all $\mathrm{j}$ and $\mathrm{k}, b_{j} \geq 0$ for all $\mathrm{i}=1,2, \ldots, \mathrm{m} .[10]$

Wolfe has suggested introducing $\mathrm{n}$ non-negative artificial variable $v_{j}$ in to the equation representing $\partial L / \partial x_{j}=0$

$\sum_{i=1}^{n} g_{i j} x_{i}+\sum_{i=1}^{m} \lambda_{i} a_{i j}+\mu_{j}+v_{j}=c_{j}$

Now, starting with an initial basic solution $\mathrm{v}=\mathrm{c}, \mathrm{s}=\mathrm{b}$ and $\mathrm{x}=0, \lambda=0$,

We maximize

$z_{v}=-\sum_{i=1}^{n} v_{i}$

Subject to

$-\mu+c+g_{i} x++a_{j} \lambda-v=0$

$A x+s=b$,

$\mu, x, s, \lambda, v \geq 0$

And the complementary slackness conditions are:

$\lambda_{i} s_{i}=0, i=1, \ldots, m$.

$\mu_{i} x_{i}=0, i=1, \ldots, n$.

With one exception, this is exactly the linear programming problem with we can solve by usual simplex method. The simplex algorithm needs to be modified to include the complementary slackness conditions. Thus, when deciding whether to introduce $s_{i}$ in the solution, it is a must first to ensure either that $\lambda_{i}$ is not in the solution or that $\lambda_{i}$ will be removed when $s_{i}$ enters. This additional check is not difficult to perform by using simplex and routine that has been successfully performed. In other words, the solution of the above system is obtained by using phase I of two-phase method, is to obtain a feasible and hence the solution does not require a consideration of phase II. The only restriction is that the condition $\lambda_{i} s_{i}=0=\mu_{i} x_{i}$ should be maintained all the time. This implies that if $\lambda_{i}$ is in the basic solution with positive value, then $s_{i}$ cannot be based with +ve value. Similarly, $\mu_{i}$ and $x_{i}$ cannot be positive simultaneously. It should be noted that phase I will end in the usual manner with the sum of the artificial variable equal to zero only if the feasible solution to the problem exist. [12]

\subsection{Algorithm for the Wolfe's method}

Step 1: First convert the inequality constraints into equations by introducing slack variables $q_{1}^{2}$ in the $i^{\text {th }}$ constraint $(\mathrm{i}=1,2 \ldots, \mathrm{m})$ and the slack variables $r_{1}^{2}$ in the $j^{t h}$ non-negativity constraint $(\mathrm{j}=1,2, \ldots, \mathrm{n})$.

Step 2: Then, construct the Lagrangian function

$L(x, q, r, \lambda, \mu)=f(x)-\sum_{i=1}^{m} \lambda_{i}\left[\sum_{i=1}^{n} a_{i l}-b_{j}+q_{i}^{2}\right]-\sum_{j=1}^{n} \mu_{j}\left[-x_{j}+r_{j}^{2}\right]$

Where $x=\left(x_{1}, x_{2}, \ldots, x_{n}\right)$,

$x=\left(x_{1}, x_{2}, \ldots, x_{n}\right)$,

$r=\left(r_{1}, r_{2}, \ldots, r_{n}\right)$,

$\lambda=\left(\lambda_{1}, \lambda_{2}, \ldots, \lambda_{n}\right)$, 
$\mu=\left(\mu_{1}, \mu_{2}, \ldots, \mu_{n}\right)$,

Differentiating L partially w.r.t. the components of $\mathrm{x}, \mathrm{q}, \mathrm{r}, \lambda, \mu$ and equating the first order partial derivatives to zero, Kuhn-Tucker conditions are obtained.

Step 3: Introduce the non-negative artificial variable $v_{j}, \mathrm{j}=1,2, \ldots, \mathrm{n}$ in the Kuhn Tucker condition

$c_{j}+\sum_{k=1}^{n} c_{j k} x_{k}-\sum_{j=1}^{n} a_{i j} \lambda_{i}+\mu_{j}=0$ for $\mathrm{j}=1,2, \ldots, \mathrm{n}$ and to construct an objective function

$z_{v}=v_{1}+v_{2}+\cdots+v_{n}$

Step 4: Obtain the initial basic feasible solution to the following linear programming problem $\min z_{v}=v_{1}+v_{2}+\cdots+$ vn. subject to the constraints

$\sum_{k=1}^{n} c_{j k} x_{k}-\sum_{k=1}^{n} c_{j k} x_{k}-\sum_{k=1}^{n} a_{i j} \lambda_{i}+\mu_{j}+v_{j}=-c_{j}$ for $(\mathrm{j}=1,2, \ldots, \mathrm{n})$

$\sum_{k=1}^{n} a_{i j} x_{j}+q_{i}^{2}=b_{j}$

Where $\mathrm{i}=(1,2, \ldots, \mathrm{m}), v_{j}, \lambda_{i}, \mu_{j}, x_{j} \geq 0(\mathrm{i}=1,2, \ldots, \mathrm{m} ; \mathrm{j}=1,2, \ldots, \mathrm{n})$

And satisfying the complementary slackness condition:

$\sum_{k=1}^{n} \mu_{j} x_{j}-\sum_{i=1}^{m} \lambda_{i} s_{i}=0$ where $\left(s_{i}=q_{i}^{2}\right) \lambda_{i} s_{i}=0$ and $\mu_{j} x_{j}=0$

$(i=1,2, \ldots, m ; j=1,2, \ldots, n)$

Step 5: Now apply 2- phase simplex method to find and optimum solution of Linear Programming problem in step 4 .The solution must satisfy the above complementary slackness condition.

Step 6: Thus the optimum solution obtained in step 5 is the optimal solution of the given Quadratic programming problem (QPP). [10]

\section{Modified simplex method development}

Simplex method is developed by Dantzig in (1947). The Simplex method provides a systematic algorithm which consists of moving from one basic feasible solution (on the vertex) to another in the prescribed manner so the value of the objective function is elicited. This procedure of jumping from vertex to vertex is repeated. If the objective function is improved at each jump, then no basis can ever be repeated and there is no need to go to back to vertex because it is already covered. Since the number of vertices is finite, the process must lead to the optimal vertex in a finite number throughout the steps.

The Simplex algorithm is an iterative (step by step) procedure for solving linear programming problems. It consists of:

i) Having a trail basic feasible solution to constraint equations.

ii) Testing whether is an optimal solution.

iii) Improving the first trial solution by a set of rules, and repeating the process till an optimal solution is obtained.

For more details [11].

\subsection{Solution for quadratic programming problem by modified simplex method:}

This section deals with the solution of the quadratic programming problem by the method exactly similar to Simplex Technique in linear programming. This method can be successfully adapted to high speed computational. We can apply this method if the constraints of the problem are linear function. i.e. our problem is of the form:

Max.z (or Min. $\mathrm{z})=\left(c^{T} x+\alpha\right)\left(d^{T} x+\beta\right)$

Subject to: 
$\operatorname{Ax}\left(\begin{array}{l}\leq \\ \geq \\ =\end{array}\right) \mathrm{b}$
$\mathrm{x} \geq 0$

i) $\quad \mathrm{A}$ is $\mathrm{m} \times \mathrm{n}$ matrix;

ii) $\quad \mathrm{x}, \mathrm{c}, \mathrm{d}$ are $\mathrm{n} \times 1$ column vectors;

iii) $\quad \mathrm{b}$ is $\mathrm{m} \times 1$ column vectors;

iv) $\quad \alpha, \beta$ are scalars and prime (T) denoted the transpose of the vector.

Here it is assumed that $\left(c^{T} x+\alpha\right)\left(d^{T} x+\beta\right)$ are positive all feasible solutions, the set of all feasible bounded, and closed convex polyhedron. Also, at least two distinct feasible solutions exist. [11]

To apply simplex process, first $\Delta_{j 1}$ and $\Delta_{j 2}$ need to be found from the coefficients of the first linear vector and second linear vector of objective function respectively, by using the following formula:

$\Delta_{j i}=c_{i j}-c_{B i} x_{i j}, \mathrm{i}=1,2 . \mathrm{j}=1,2, \ldots, \mathrm{m}+\mathrm{n}$

$z_{1}=c_{B 1} v_{B}+\alpha$

$z_{2}=c_{i j} v_{B}+\beta$

$z=z_{1} \times z_{2}$

And compute $\mu_{j}=\min \left[{ }^{v_{B}} / x_{j}\right]$ for non-basis vector, in this approach the formula is defined to find $\Delta_{j}$ from $\Delta_{j 1}, \Delta_{j 2}, z_{1}, z_{2}$ and $\mu_{j}$ following:

$\Delta_{j}=z_{1} \Delta_{j 2}+z_{2} \Delta_{j 1}+\mu_{j} \Delta_{j 1} \Delta_{j 2}$.

\subsection{Algorithm for modified simplex method}

An algorithm for solve QPP by modified simplex method can be summarized as following:

Step 1: Write the standard from of the problem, by introduction slack and artificial variables to constraints, and write starting simplex table.

Step 2: Calculate the $\Delta_{j}$ by the following formula. $\Delta_{j}=z_{1} \Delta_{j 2}+z_{2} \Delta_{j 1}+\mu_{j} \Delta_{j 1} \Delta_{j 2}$, then write it in the starting simplex table

Step 3: Find the solution by using simplex process.

Step 4: Check the solution for feasibility in slep3, if it is feasible then go to step5, otherwise use dual simplex method to remove infeasibility.

Step 5: Check solution for optimality if all $\Delta_{j} \geq 0$, then the solution is optimal, otherwise go to step 3.

\section{Mathematical form of QFPP}

The mathematical form of this type of problems is given as follows:

$\max . z=\frac{c^{T} x+\frac{1}{2} x^{T} G_{1} x}{d^{T} x+\frac{1}{2} x^{T} G_{2} x}$

Subject to:

$$
\begin{aligned}
A x\left[\begin{array}{l}
\geq \\
\leq \\
=
\end{array}\right] b \\
x \geq o
\end{aligned}
$$

Where $G_{1}$ and $G_{2}$ are $(n \times n)$ matrix of coefficients with $G_{1}, G_{2}$ are symmetric matrixes. All vectors are assumed to be column vectors unless transposed $(T)$. Where $x$ is an $n$-dimensional vector of decision variables, $C$ is the $n$-dimensional vector of constants, $b$ is $n$-dimensional vector of constants.

In this work the problem that has objective function is tried to be solved as the form.

$\max . z=\frac{\left(c_{1}^{T} x+\frac{1}{2} x^{T} G_{1}^{1} x\right)\left(c_{1}^{T} x+\frac{1}{2} x^{T} G_{1}^{2} x\right)}{\left(d_{1}^{T} x+\frac{1}{2} x^{T} G_{2}^{1} x\right)\left(d_{2}^{T} x+\frac{1}{2} x^{T} G_{2}^{2} x\right)}$ 
Subject to:

$$
\begin{aligned}
& A x\left[\begin{array}{l}
\geq \\
\leq \\
=
\end{array}\right] b \\
& x \geq 0
\end{aligned}
$$

Where $G_{1}^{1}, G_{1}^{2}, G_{2}^{1}$ and $G_{2}^{2}$ are $(n \times n)$ matrix of coefficients with $G_{1}^{1}, G_{1}^{2}, G_{2}^{1}$ and $G_{2}^{2}$ are symmetric matrixes. All vectors are assumed to be column vectors unless transposed $(T)$. where $x$ is an $n$-dimensional vector of decision variables, $c$, $d$ are the $n$-dimensional vector of constants, $b$ is $n$-dimensional vector of constants.

\section{Formulation of QFPP function}

$\max . z=\frac{z_{1}}{z_{2}}$

Where $z_{1}=c^{T} x+\frac{1}{2} x^{T} G_{1} x=\left(c_{1}^{T} x+\frac{1}{2} x^{T} G_{1}^{1} x\right)\left(c_{1}^{T} x+\frac{1}{2} x^{T} G_{1}^{2} x\right)$

$z_{2}=d^{T} x+\frac{1}{2} x^{T} G_{2} x=\left(d_{1}^{T} x+\frac{1}{2} x^{T} G_{2}^{1} x\right)\left(d_{2}^{T} x+\frac{1}{2} x^{T} G_{2}^{2} x\right)$

And supposed $z_{1}^{1}=\left(c_{1}^{T} x+\frac{1}{2} x^{T} G_{1}^{1} x\right)$

$z_{1}^{2}=\left(c_{1}^{T} x+\frac{1}{2} x^{T} G_{1}^{2} x\right)$

$z_{2}^{1}=\left(d_{1}^{T} x+\frac{1}{2} x^{T} G_{2}^{1} x\right)$

$z_{2}^{2}=\left(d_{2}^{T} x+\frac{1}{2} x^{T} G_{2}^{2} x\right)$

And $\max . z_{1}=\varphi_{1}$

$\max . z_{2}=\varphi_{2}$

Finally $\max . z=\frac{\varphi_{1}}{\varphi_{2}}$. where $\varphi_{2} \neq 0$

And $\frac{\varphi_{1}}{\varphi_{2}}$ the values of objective functions.

\section{Algorithm for QFPP}

The value of $z_{1}$ and $z_{2}$ found by Wolfe's Algorithm in section 4.1as well as modified simplex algorithm in section 5.2 in this paper.

\section{Numerical examples and results}

In this section we tested several numerical examples but only two of them are presented. Consider the following Quadratic Fractional Programming Problem as:

\section{Example 1:}

Maximize $\mathrm{Z}=\frac{\left(x_{2}+1\right)\left(x_{1}+x_{2}+3\right)}{\left(x_{1}+4\right)\left(x_{1}+x_{2}+2\right)}$

Subject to:

$$
\begin{gathered}
-x_{1}+x_{2} \leq 1 \\
x_{1}+2 x_{2} \leq 7 \\
x_{1}, x_{2} \geq 0
\end{gathered}
$$

Solution: After finding the values of each individual objective functions of example 1 by Wolfe's method, the following result is found.

$x_{1}=5 / 3, x_{2}=8 / 3, \max . z_{1}=176 / 3, \max . z_{2}=323 / 3, \max . \mathrm{z}=0.544$.

When the same example is solved by modified simplex method produces the same result as Wolfe's method see below. $x_{1}=5 / 3, x_{2}=8 / 3, \max . z_{1}=176 / 3, \max . z_{2}=323 / 3, \max . \mathrm{z}=0.544$. 


\section{Example 2:}

Maximize $\mathrm{Z}=\frac{\left(x_{1}+2\right)\left(x_{1}+x_{2}+1\right)}{\left(x_{1}+2 x_{2}+3\right)\left(x_{2}+3\right)}$

Subject to:

$$
\begin{gathered}
4 x_{1}+2 x_{2} \leq 8 \\
x_{1}+2 x_{2} \leq 6 \\
x_{1}, x_{2} \geq 0
\end{gathered}
$$

Solution: The results of each individual objective functions of example 2 by Wolfe's method the following result is found.

$x_{1}=2 / 3, x_{2}=2.6, \max . z_{1}=11.373, \max . z_{2}=49.649, \max . \mathrm{z}=0.229$.

And the results of above example when solved by modified simplex method almost it same Wolfe's method result are shown below.

$x_{1}=0.7, x_{2}=2.6, \max . z_{1}=11.61, \max . z_{2}=49.84, \max . \mathrm{z}=0.232$.

\section{Comparison of the numerical results is shown in the following table}

\begin{tabular}{|c|c|c|}
\hline Examples & Wolfe's Methods & Modified Simplex Method \\
\hline \multirow{2}{*}{ Ex.1 } & $x_{1}=5 / 3, x_{2}=8 / 3, \max . z_{1}=176 / 3$, & $x_{1}=5 / 3, x_{2}=8 / 3, \max . z_{1}=176 / 3$, \\
& $\max . z_{2}=323 / 3, \max . \mathrm{z}=0.544$. & $\max . z_{2}=323 / 3$, \\
& $\max . \mathrm{z}=0.544$. \\
\hline \multirow{2}{*}{ Ex.2 } & $x_{1}=2 / 3, x_{2}=2.6, \max . z_{1}=11.373$, & $x_{1}=0.7, x_{2}=2.6, \max . z_{1}=11.61$ \\
& $\max . z_{2}=49.649$, & $\max . z_{2}=49.84$, \\
$\max . \mathrm{z}=0.229$ & $\max . \mathrm{z}=0.232$. \\
\hline
\end{tabular}

In the above table, it is notice that value of objective function in ex. (1) when using Wolfe's Method, the same results are obtained when it solved by the Modified simplex method. However ex. (2) max. $\mathrm{z}=0.229$ when is solved by Wolfe's method is lower than the result $\max . \mathrm{z}=0.232$ when it solved by modified simplex method. Those results are promising and interesting as it being addressed for the first time.

\section{Conclusion}

This paper used the Wolfe's method and modified simplex method to found the maximum value of QFPP, The comparisons of these methods are based on the value of the objective function, the study found that max.z resulted with better, larger value with modified simplex method than the max.z value that found with the Wolf's methods when both used to produce the optimal solution of the QFPP. Therefore, this paper recommends that modified simplex method is superior in addressing QFPP.

\section{References}

[1] A. Antoniou, Wu-Sheng Lu, Practical Optimization: Algorithms and Engineering Applications, Department of Electrical and Computer Engineering University of Victoria, Canada. (2007). 675.

[2] A. Beck, A. Ben-Tal and M. Teboulle Finding A Global Optimal Solution For A Quadratically Constrained Fractional Quadratic Problem With Applications To The Regularized Total Least Squares, Society for Industrial and Applied Mathematics Siam J. Matrix Anal. Appl.Vol. 28, No. 2, (2006), 425-445.

[3] M, Hasan. (July), A Technique for Solving Special Type Quadratic Programming Problems, Department of Mathematics, University of Dhaka, Dhaka-1000, Bangladesh, Dhaka Univ. J. Sci. 60(2): (2012), 209-215.

[4] M. Borza, A. Rambely, M. Saraj, Solving Linear Fractional Programming Problems with Interval Coefficients in the Objective Function. A New Approach, Department of Mathematics, Faculty of Mathematical Sciences \& Computer, Shahid Chamran University, Ahvaz-Iran, Applied Mathematical Sciences, Vol. 6, 2012, no. 69, (2012), 3443 - 3452.

[5] M. Biggs, Nonlinear Optimization with Financial Applications, Kluwer Academic Publishers, England. (2005),41.

[6] M. Fukushima, Sh. Hayashi, , Quadratic Fractional Programming Problems with Quadratic Constraints, Department of Applied Mathematics and Physics, Graduate School of Informatics, Kyoto University. (2008).

[7] M.Vankova, Algorithms for the Solution of the Quadratic Programming Problem, the Faculty of Science at the University Of Port Elizabeth. (2004), 36.

[8] P. Amaral, I. Bomze, J. Judice, Copositivity and Constrained Fractional Quadratic Problems, www.optimization online.org/ DB_FILE /2010 /06/2641.pdf, (2011). 
[9] P. Wolfe, the Simplex Method for Quadratic Programming Economics, Vol.27,1959, 382-398.

[10] R. Singh, Optimization Methods and Quadratic Programming, Department of Mathematics National Institute of Technology, Rourkela Odisha, India, (2012), 28.

[11] S. Sharma, Operation Research, Kedar Nath Ram Nath BCO. Meerut, India. (1988)

[12] S, Sharma, Nonlinear and Dynamic Programming, Kedar Nath Ram Nath and CO, Meerut. (1980) 